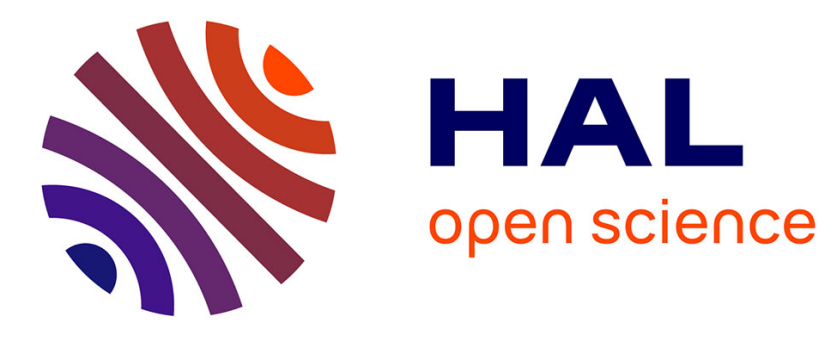

\title{
SnSe: Breakthrough or Not Breakthrough?
}

Christophe Candolfi, Dorra Ibrahim, Jean-Baptiste Vaney, Selma Sassi, Philippe Masschelein, Anne Dauscher, Bertrand Lenoir

\section{To cite this version:}

Christophe Candolfi, Dorra Ibrahim, Jean-Baptiste Vaney, Selma Sassi, Philippe Masschelein, et al.. SnSe: Breakthrough or Not Breakthrough?. Sergey Skipidarov; Mikhail Nikitin. Novel Thermoelectric Materials and Device Design Concepts, Springer International Publishing, pp.23-46, 2019, 978-3-03012056-6. 10.1007/978-3-030-12057-3_2 . hal-03027239

\section{HAL Id: hal-03027239 \\ https://hal.science/hal-03027239}

Submitted on 27 Nov 2020

HAL is a multi-disciplinary open access archive for the deposit and dissemination of scientific research documents, whether they are published or not. The documents may come from teaching and research institutions in France or abroad, or from public or private research centers.
L'archive ouverte pluridisciplinaire HAL, est destinée au dépôt et à la diffusion de documents scientifiques de niveau recherche, publiés ou non, émanant des établissements d'enseignement et de recherche français ou étrangers, des laboratoires publics ou privés. 


\title{
SnSe: breakthrough or not breakthrough?
}

Christophe Candolfi*, Dorra Ibrahim, Jean-Baptiste Vaney, Selma Sassi, Philippe Masschelein, Anne Dauscher, Bertrand Lenoir

Institut Jean Lamour, UMR 7198 CNRS - Université de Lorraine, 2 allée André Guinier, BP 50840, 54011 Nancy, France

${ }^{*}$ Corresponding author: christophe.candolfi@univ-lorraine.fr

\begin{abstract}
The simple binary semiconductor SnSe has not been for long considered as a promising thermoelectric material compared to PbSe. For this reason, its thermoelectric properties have been largely overlooked over the past decades. This compound came back to the forefront of research in thermoelectricity due to the very high $Z T$ values reported recently in single crystals. This announcement has been the starting point of a renewed interest leading to a wealth of experimental and theoretical studies in the past few years with the aim of better understanding its physical properties and optimizing its thermoelectric performances in both $p$ - and $n$-type samples. Here, we review the progress of research on the transport properties of both single-crystalline and polycrystalline SnSe, highlighting in particular the controversy regarding the thermal properties of single crystals and the important points that
\end{abstract}


remain to be investigated to determine whether or not the (re)discovery of SnSe may be considered as a breakthrough in thermoelectricity.

Keywords: thermoelectric; chalcogenide; SnSe; semiconductor; thermal conductivity

\section{Introduction}

The design of highly-efficient thermoelectric materials represents a formidable challenge. Such a compound should indeed strike a subtle balance between electronic and thermal properties [1-3]. A high thermopower $\alpha$ and low electrical resistivity $\rho$ typical of heavily-doped semiconductors should be reconciled with low total thermal conductivity $\kappa$, usually seen in glassy systems, in order to maximize the dimensionless thermoelectric figure of merit $Z T=\alpha^{2} T / \rho \kappa=P T / \kappa$ at an absolute temperature $T$ [1-3]. Adding another degree of complexity, these mutually incompatible properties cannot be optimized independently since they are all interdependent through the charge carrier concentration. Most of the best thermoelectric materials possess favourable energy band structure with multiple valleys that helps to achieve high power factors $P$ [1-3]. Concomitantly, they also harbour specific crystallographic features that strongly limit the ability of acoustic phonons to propagate such as, for instance, localized lattice vibrations ("rattling modes") or chemical bonds that vibrate anharmonically [4-8]. Additional geometric architecturing such as nanostructuring or inducing nanoscale domains through controlled synthetic conditions represents another 
strategy to limit the heat transport and thus, achieve better thermoelectric performances [912].

For decades, the family of lead telluride ( $\mathrm{PbTe})$ compounds and its selenide analogue PbSe have been the thermoelectric materials of choice for operating at medium temperatures (typically near $500 \mathrm{~K}$ ) [1-3]. These semiconductors gather many important characteristics to be a prospective area of research for achieving high thermoelectric performances [1-3]. Despite a simple cubic rock-salt crystal structure, the lattice thermal conductivity is very low due to significant anharmonicity in the lattice that originates from a strong anharmonic coupling between transverse optic modes and longitudinal acoustic modes, as shown by neutron spectroscopy techniques [13-15]. The electronic band structure with non-parabolic bands and multiple valleys is the key ingredient that explains the good thermoelectric properties achieved by proper doping. In comparison to other chalcogenide semiconductors, PbTe-based compounds have received significant attention in the 1960s and 1970s and are still the subject of numerous studies nowadays [1-3].

Tin selenide SnSe is another representative of the vast family of chalcogenide semiconductors whose basic crystallographic and physical properties have also been investigated in these decades [16-18]. Although chemically similar to PbTe, SnSe crystallizes with a distorted lattice described in the orthorhombic space group Pnma [19-21]. In retrospective, it is perhaps not so surprising that this compound has received less attention in this period. Its orthorhombic crystal structure gives rise to anisotropic transport properties, even in polycrystalline samples. In addition, the number of extrinsic dopants that can be introduced in SnSe seemed more restricted than in PbTe-based compounds. For 
these reasons, significant efforts were devoted to optimize the thermoelectric properties of $\mathrm{PbTe}$ and its derivatives.

In 2014, this forgotten, "ugly-duckling" compound made a remarkable comeback to the forefront of research in thermoelectricity with the announcement of record-breaking ZT values of up to 2.6 at $900 \mathrm{~K}$ in single crystals $[22,23]$. What was even more surprising was the fact that such high values have been achieved in pristine single crystals while high ZT values are usually reached once a careful optimization of the electronic and thermal properties has been realized. Another striking outcome of this report was the very low lattice thermal conductivity measured at high temperatures ranking this compound among the best thermal insulators known to date [22]. Such extremely rare combination of transport properties raised a number of questions: Can one optimize even further the thermoelectric performances of single-crystalline SnSe through doping? Is it possible to achieve similar ZT values in polycrystalline samples more suitable for integration in thermoelectric generators? What microscopic mechanisms impede so efficiently the propagation of the heat-carrying acoustic phonons in such a simple crystal structure? In addition to these important questions of both practical and fundamental interest, the difference between the thermal conductivity measured in this study [22] and that reported in the 1960s [16-18] has rapidly become another source of interrogation and debate within the thermoelectric community. Although consensual answers to some of these questions are yet to be obtained, this announcement stirred up strong interest to revisit in detail the physical properties of this compound by modern techniques. 
In this chapter, we will provide a brief review of the accumulated knowledge acquired on this compound based on the experimental and theoretical results obtained over the last few years. We will focus more particularly on the low-temperature allotrope of SnSe referred to as the $\alpha$ form which shows the best thermoelectric performances. Our goal is to highlight the important structural and chemical aspects that influence its transport properties and on the strategies employed to optimize its thermoelectric performances. As excellent reviews on this compound and, more generally, on tin chalcogenides already exist [24-26], we will only focus on the main aspects and issues that recently emerged and remain to be elucidated in future studies.

\section{Crystal structure, synthesis and defect chemistry}

\subsection{Crystal structure}

At room temperature, SnSe adopts an orthorhombic crystal structure (space group Pnma, No. 62; $a=11.37 \AA, b=4.19 \AA$ and $c=4.44 \AA$ at $300 \mathrm{~K}$ ) which can be thought of as a three-dimensional distortion of the rock-salt cubic structure of PbTe (Figure 1) [19-21]. The basic building block is composed of two-atom-thick SnSe slabs that run along the $b-c$ plane. These slabs are bound by weak Sn-Se bonding along the $a$ axis and are corrugated, creating zig-zag chains when the structure is projected along the $b$ direction (see Figure 1). Interestingly, this creased honeycomb structure is similar to that of black phosphorus whose 
sheets, called phosphorene, are currently attracting attention for fast-response electronic and photonic devices [27].
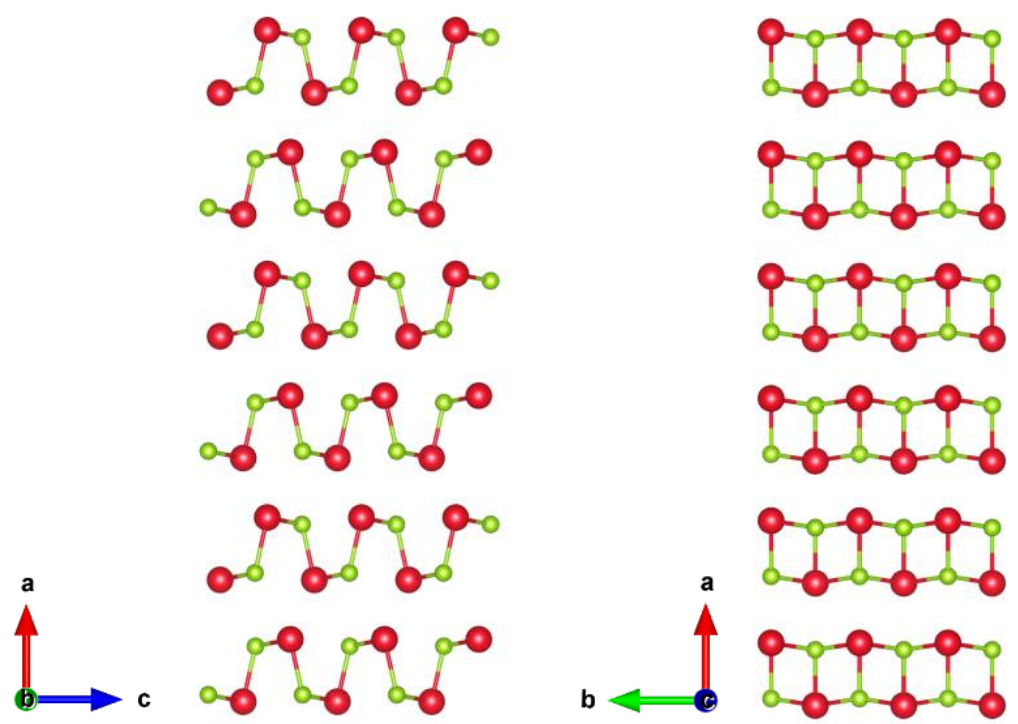

Fig. 1 Projection of the crystal structure of SnSe (space group Pnma, No. 62) along the $b$ and $c$ axis. The Sn and Se atoms are in red and green, respectively.

Upon warming, SnSe undergoes a displacive structural transition near $800 \mathrm{~K}$ which leaves the orthorhombic symmetry of the unit cell unchanged $[22,28]$. The high-temperature allotrope, referred to as $\beta$-SnSe, is described in the space group Cmcm (No. 63) with roomtemperature lattice parameters $a=4.31 \AA, b=11.71 \AA$ and $c=4.32 \AA[22,28]$. Other allotropes have been identified at high pressures with structural modifications above $27 \mathrm{GPa}$ that gives rise to a topologically-non-trivial semi-metallic state and to superconductivity at $39 \mathrm{GPa}$ with a critical temperature of $3.2 \mathrm{~K}$ [29-37]. The $\alpha$-to- $\beta$ transition, which has been directly imaged by high-resolution transmission electron microscopy [22], leaves clear signature on 
both the transport and thermodynamic properties. As we shall see below, the anisotropic crystal structure of SnSe results in a significant anisotropy in the transport properties, should the samples be in single-crystalline or polycrystalline form.

\subsection{Synthesis of polycrystalline and single-crystalline SnSe}

Since elemental Sn and Se are commercially available with very low impurity levels, both highly-pure polycrystalline and single-crystalline samples can be easily synthesized. Large polycrystalline specimens can be obtained by direct melting the powders or shots of the elements at high temperatures in sealed silica ampoules. Unlike other thermoelectric materials for which such technique does not necessarily yield homogeneous ingots due to their inherent defect chemistry, polycrystalline SnSe samples appear to be well homogeneous.

Large single crystals can be synthesized by more complex growth techniques. While early studies on SnSe used vapour phase techniques in sealed silica tubes [16-18], another elegant technique is the Bridgman technique. This method implies to pull the ampule with a carefully shaped end through a large temperature gradient with the highest temperature above the melting point of the compound. The crystal then solidifies from a seed at the shaped end of the ampoule and progressively forms along its length. This process has been carried out successfully in both horizontal and vertical configurations to obtain large, centimetre-sized single crystals of SnSe. However, one major issue regarding the growth of single-crystalline SnSe is related to the phase transition undergone near $800 \mathrm{~K}$, that is, below 
the melting point of this compound $(1134 \mathrm{~K})$. Together with the high vapour pressure of Se, the volume increase across the $\beta$-to- $\alpha$ transition (around 2.5\%) can lead to breakage of the ampoule during the process. This problem can be mitigated by sealing the first silica ampoule into a second one to prevent loss of Se vapour and oxidation of the elements. The single crystals obtained by such techniques have typically centimetre-size lateral dimensions and can be easily cleaved along the $a$ and $c$ axes [22,38-54]. Of note, other techniques related to hydro- and solvothermal syntheses have been also successfully used to obtain singlecrystalline plates or nanobelts [55,56]. Obtaining large crystals is a necessary prerequisite to study in detail the components of the transport properties tensors and allows for more sophisticated spectroscopic techniques to be employed.

\subsection{Defect chemistry}

As many thermoelectric materials [1-3], defect chemistry plays an important role in determining the transport properties of SnSe. Although this predisposition to defects is not as critical in SnSe as in the well-known $\mathrm{Bi}_{2} \mathrm{Te}_{2}$-based alloys for instance, their presence can nevertheless result in single crystals with physical properties varying along the growth direction. In particular, such variations can be problematic when combining transport properties measured on samples taken at different positions in the crystal to calculate the ZT values.

Pioneering works on single crystals of SnSe grown by vapour techniques have probed the full range of carrier concentrations showing that only $p$-type crystals can be 
obtained with hole concentrations varying between $3 \times 10^{15}$ and $2 \times 10^{18} \mathrm{~cm}^{-3}$ at $300 \mathrm{~K}$ [16-18]. The hole concentration strongly depends on the details of the synthetic procedure and possible subsequent annealing step employed (a process referred to as saturation annealing). Although this $p$-type nature was supposed to be due to an excess of Se atoms [16-18], it was not clear at that time whether Sn vacancies, antisite defects or both types of defects were responsible for this behaviour. Furthermore, laboratory structural and chemical probes such as X-ray diffraction or energy-dispersive spectroscopy are not sensitive enough to discriminate between different amounts and types of defects. The determination of the main type of defects has been recently addressed by detailed electronic band structure calculations [57]. These theoretical studies are consistent with native $p$-type doping primarily due to Sn vacancies which are energetically favoured over antisite defects under both Sn- and Se-rich conditions. Another possible source of extrinsic $p$-type doping has been recently advanced based on the presence of $\mathrm{SnSe}$ microdomains in single crystals grown under various experimental conditions [58]. These inhomogeneities are believed to yield interfacial charge transfer to occur from SnSe to SnSe2 resulting in an overall hole-doped SnSe matrix. Yet, as mentioned by the authors, the high cooling rate employed during the growth process is likely the key parameter that leads to the nucleation of this secondary phase. Several other recent studies performed on single crystals have indeed not reported the presence of such domains while these pristine crystals remain intrinsically hole-doped. 


\section{Electronic band structure and electronic properties}

\subsection{Electronic band structure of $\alpha$-SnSe}

Significant efforts have been devoted to determine both theoretically and experimentally the main features of the electronic band structure of SnSe that govern its transport properties. While some dispersion in the computed band gap exists in the literature due to the details of the calculations and the method used, most of the studies indicate a band gap on the order of $0.6-0.9 \mathrm{eV}$ [22,43,59-72]. This value is consistent with the experimental band gap values of 0.86 and $0.92 \mathrm{eV}$ obtained by optical absorption spectroscopy $[22,54]$.

The band gap is indirect with a valence band maximum (VBM) lying along the $\Gamma-\mathrm{Z}$ direction while the conduction band minimum $(\mathrm{CBM})$ is located along the $\Gamma-Y$ direction (Figure 2) [60]. The overall structure of the valence and conduction bands is rather complex with several maxima and minima, respectively, close to each other in energy. In particular, the highest VBM shows a "pudding-mould-like" shape, that is, a band that shows a dispersive part and a flatter portion nearby [60]. Such a shape has in particular been proposed to be beneficial for achieving good thermoelectric performances [73]. Furthermore, a second valence band maximum lies just below the main valence band maximum. 


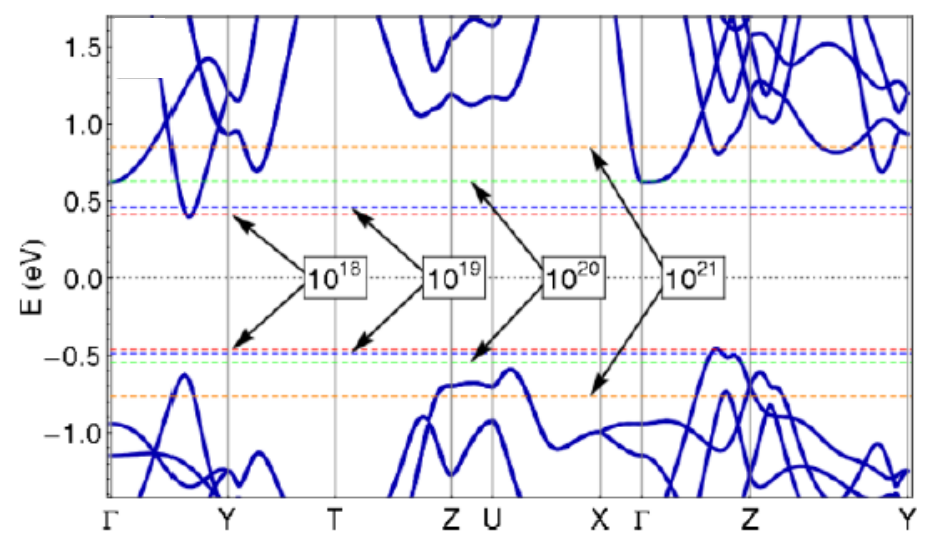

Fig. 2 Electronic band structure along high-symmetry directions of the low-temperature structure of SnSe (space group Pnma, No 62) calculated by the Korringa-Kohn-Rostoker method in Ref [60]. The dashed horizontal lines correspond to various hole and electron concentrations used to illustrate the bands that participate to the conduction at these concentrations. The pudding-mould-like band is located along the $\Gamma-\mathrm{Z}$ direction. Reproduced Figure 2 (panel a) with permission from Kutorasinski et al. [60]. Copyright 2018 by the American Physical Society. DOI: 10.1103/PhysRevB.91.205201.

The indirect nature of the band gap implies anisotropic density-of-states effective masses that differ significantly between the three crystallographic axes. The density-of-states effective mass ellipsoids were calculated to be equal to $0.74 \mathrm{~m}_{0}, 0.31 \mathrm{~m}_{0}, 0.16 \mathrm{~m}_{0}$ ( $\mathrm{m}_{0}$ is the bare electron mass) for the highest VBM, and 2.40mo, $0.11 \mathrm{~m}_{0}, 0.15 \mathrm{~m}_{0}$ for the lowest CBM along the $a, b$ and $c$ axes, respectively [59]. The second VBM shows similar values along the $a$ and $c$ directions $(0.90 \mathrm{~m} 0$ and $0.15 \mathrm{~m} 0$, respectively) while it differs more significantly along the $b$ 
direction $(0.12 \mathrm{~m} 0)$ [59]. The presence of multiple local extremum near the band edges is an important aspect to understand the good electronic properties achieved in this compound as we will see below.

Based on the computed band structure, the electrical conductivity and thermopower have been computed using the Boltzmann transport approach for a wide range of temperatures and $p$ - and $n$-type doping levels [60]. The results evidenced significant anisotropy in the transport properties between the different crystallographic axes, as expected for such layered crystal structure. The anisotropy depends on the carrier concentration and while at relatively high concentrations the thermopower shows only little variations between the three directions, more significant differences may appear at carrier concentrations on the order of $10^{17} \mathrm{~cm}^{-3}$. One of the main results of these calculations is related to the prediction that $n$-type SnSe should potentially exhibit higher thermoelectric performances with respect to $p$-type analogues [60].

\subsection{Angle-resolved photoemission spectroscopy}

Because the details of the electronic band structure can depend on the particular computational method used, direct experimental probes of the electronic band structure are particularly relevant to unveil its main characteristics in both pristine and hole-doped crystals. The growth of high quality, large single crystals and the ease with which they can be cleaved make angle-resolved photoemission spectroscopy (ARPES) a powerful tool for such investigations. The experimental investigation of the valence band structure of SnSe by 
this technique has been recently addressed by several groups as a function of the doping level (undoped and Na-doped single crystals) and temperature [27,58,74-76]. Probably due to the low doping level studied, the presence of $\mathrm{Na}$ had little influence on the main characteristics of the band structure of SnSe [76]. Regarding pristine SnSe, all these studies consistently evidenced the presence of highly anisotropic valence bands with multiple valleys in agreement with available calculated band structures (Figure 3).
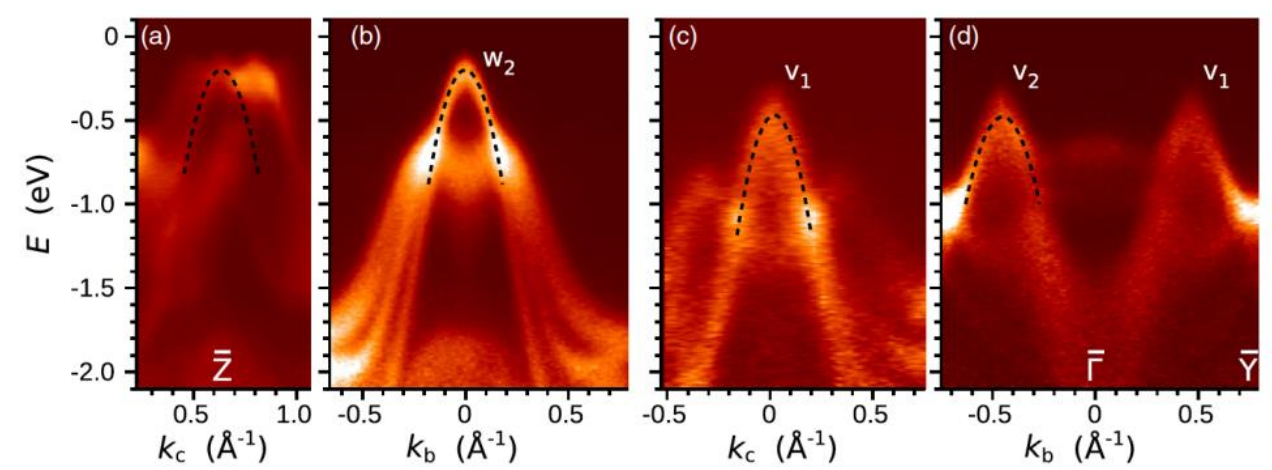

Fig. 3 Energy dispersion of the highest pockets in the valence bands of SnSe measured by

ARPES in the vicinity of the $Z$ (panels a and b) and $Y$ (panels $c$ and $d$ ) points which correspond to two perpendicular directions parallel to the layers. The overlaid dashed black curves represent fits using parabolic dispersions to estimate the effective masses.

Reproduced Figure 4 with permission from Pletikosić et al. [27]. Copyright 2018 by the American Physical Society. DOI: 10.1103/PhysRevLett.120.156403. 
The density-of-states effective masses have been derived from the collected ARPES $k$ $E$ maps along the main directions $(\Gamma-\mathrm{X}, \Gamma-\mathrm{Y}$ and $\Gamma-\mathrm{Z})$. The results obtained along the $\Gamma-\mathrm{Z}$ and $\Gamma-Y$ directions indicated effective masses on the order of $0.2 m_{0}$ along the $b$ and $c$ axes for both directions. The values along the $a$ axis are slightly higher as predicted theoretically. These results are thus consistent with each other's and in line with the predicted values. Among these studies, one of them [76] has nevertheless found values significantly higher than those obtained in other studies by one order of magnitude (effective masses of up to $2.23 m_{0}$ ) even at the lowest temperature probed. This study also evidenced that the effective masses tend to decrease with increasing temperature up to $600 \mathrm{~K}$.

These results help to understand the beneficial role of this multivalley band structure on the $Z T$ values. Indeed, the overall $Z T$ values along the $c$ axis of the crystal structure is related to band parameters through the following relation [77]

$$
Z T \propto \sum_{i} \gamma_{i} \tau_{i} \sqrt{\frac{m_{a i}^{*} m_{b i}^{*}}{m_{c i}^{*}}}
$$

where $m_{a i}^{*}, m_{b i}^{*}$ and $m_{c i}^{*}$ are the density-of-states effective masses along the $a, b$ and $c$ axis of the band $i, \tau_{i}$ is the relaxation time of the charge carriers along the transport direction for the band $i$ and $\gamma_{i}$ is the degeneracy of the band $i$ which is equal to two in SnSe. This simple formula explains, for instance, the poorer values observed along the $a$ axis. In this direction, the $Z T$ values are proportional to the coefficient $\sqrt{\frac{m_{b i}^{*} m_{c i}^{*}}{m_{a i}^{*}}}$ which is significantly smaller due 
to the less dispersive valence bands, resulting in higher density-of-states effective mass $m_{a i}^{*}$ compared to $m_{b i}^{*}$ and $m_{c i}^{*}$. Hence, these experimental results evidence the beneficial contribution of several valence bands to the transport properties and provide an insightful guidance for developing relevant transport models to describe their variations with the hole concentration (in the so-called Pisarenko-Ioffe plot).

\subsection{Electronic transport properties of pristine and doped single-crystalline SnSe}

Because of the inherent defect chemistry of SnSe, the hole concentration of the synthesized single crystals can vary from sample to sample. Since the discrimination of such small amounts of defects is very difficult, the most effective metric is the characterization of its electrical properties and notably, its carrier concentration. Pristine single-crystalline SnSe has been always obtained so far as a lightly-doped p-type semiconductor [22,38-54]. This state is reflected by the large electrical resistivity and thermopower values measured with hole carrier concentration of the order of $10^{17} \mathrm{~cm}^{-3}$ at $300 \mathrm{~K}$. As expected from its orthorhombic crystal structure, the transport properties are anisotropic and their study in single crystalline specimens requires a careful examination along the three crystallographic axes. Due to the low hole concentrations, the electrical resistivity values are typically on the order of few $\Omega . \mathrm{m}$ at $300 \mathrm{~K}$ and show a semiconducting behaviour with increasing temperature. Concomitantly, the thermopower can reach values as high as $800 \mu \mathrm{V} . \mathrm{K}^{-1}$ at 300 $\mathrm{K}[22,38-54]$. While the anisotropy remains moderate in the thermopower values, the electrical resistivity is more anisotropic following the sequence $\rho_{\mathrm{a}}>\rho_{\mathrm{c}}>\rho_{\mathrm{b}}$ along the $a, b$ and 
$c$ crystallographic axes. However, this is not to say that the thermopower cannot show some significant anisotropy as predicted theoretically [60] and demonstrated experimentally [42].

Only few studies on doped single crystals have been undertaken so far $[38,43,45,46,78-80]$. While substitutions of $\mathrm{Na}, \mathrm{Ag}$ and Bi for Sn have been considered, only the solid solution SnSe-SnS has been considered as a mean to substitute on the Se site. Percentages as low as 3 at $\% \mathrm{Na}$ are sufficient to significantly enhance the hole concentrations with maximum values higher than $10^{19} \mathrm{~cm}^{-3}$ at $300 \mathrm{~K}[38,43,45]$. Although this increase in the hole concentration induces a decrease in the thermopower values, the net result of the dopants is positive with an overall increase in the power factor at high temperatures $[38,43,45]$. Introduction of similar amount of Ag acts similarly with similar trends in the electrical properties as a function of the Ag content $[43,46]$. Intriguingly, the substitution of Sn by 6 at $\%$ of Bi switches the electrical conduction to $n$-type over the whole temperature range covered (300-800 K) [78]. With power factors that rival those obtained in p-type samples, these results show that good thermoelectric performances can be equally achieved in both $n$ - and $p$-type crystals. Without exceptions, for all these dopants, the best thermoelectric performances have been achieved along the $b$ axis as in pristine single crystals $[38,43,45,46,78-80]$. Because electronic band structure calculations have indicated that better performances could be achieved in n-type SnSe [60], further studies on single crystals with dopants known to induce an $n$-type conduction such as Br for instance (see below) would be worthwhile. 


\subsection{Electronic transport properties of pristine and doped polycrystalline SnSe}

Although the study of single crystals is illuminating in determining the basic physical mechanisms governing the transport properties and their possible evolution as a function of the doping level, the carefully-controlled environment required for their growth and their mechanical properties make them poor candidates for direct integration in thermoelectric generators. The assessment of the thermoelectric performances in polycrystalline samples thus rapidly became a central topic within the thermoelectric community.

The first reports on these aspects $[81,82]$ have demonstrated that pristine SnSe behaves as a lightly-doped $p$-type semiconductor with values of the electrical resistivity and thermopower in line with those achieved in single crystalline samples (Figure 4). The anisotropy seen in single crystals is not fully suppressed and survives in polycrystals. The transport properties thus need to be measured both parallel and perpendicular to the pressing direction to estimate correctly the $Z T$ values. 

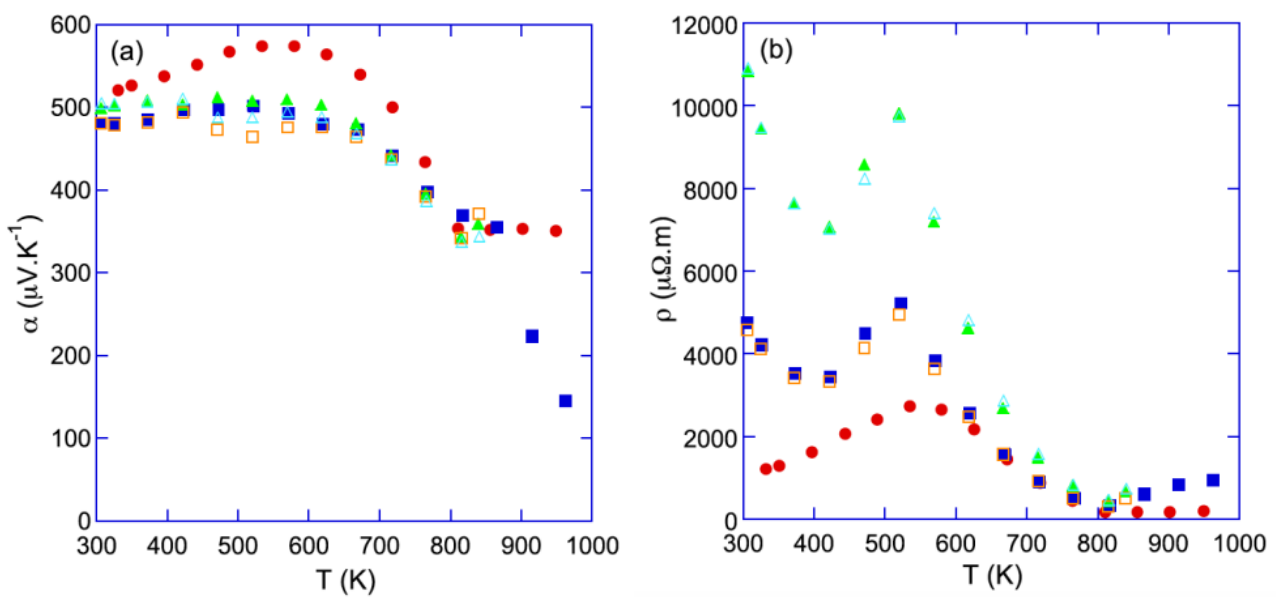

Fig. 4 Temperature dependence of the (a) thermopower and (b) electrical resistivity of undoped polycrystalline SnSe measured parallel (triangle symbols) and perpendicular (square symbols) to the pressing direction. The measurements have been performed on two different samples showing good reproducibility (distinguished by filled and open symbols). The average value of the data measured on single-crystalline SnSe [22] (filled circle symbols) have been added for comparison. Material reproduced from Sassi et al. [81], Appl. Phys. Lett., 104, 212105 (2014).

The most surprising result evidenced by these investigations [81,82], and widely confirmed in following studies, is the higher thermal conductivity values measured with respect to those reported in single crystals. Even though the values reached at high temperatures are very low for a compound with such a simple crystal structure (on the order of $0.5 \mathrm{~W} \cdot \mathrm{m}^{-1} \cdot \mathrm{K}^{-1}$ at $\left.800 \mathrm{~K}[81,82]\right)$, the difference between polycrystalline and singlecrystalline samples is particularly unexpected since the presence of grain boundary 
scattering in the former should in principle result in lower values. The origin of this discrepancy will be specifically addressed in detail below. In pristine polycrystalline samples, maximum $Z T$ values of 0.5 have been achieved at $823 \mathrm{~K}[81,82]$. Noteworthy, the orthorhombic-to-orthorhombic structural transition undergone by SnSe near this temperature leads to significant loss of Se and to irreversible plastic deformation of the samples [81]. This clearly precludes any use of this compound at temperatures above $800 \mathrm{~K}$, a conclusion further corroborated by the rapid degradation at temperatures above $873 \mathrm{~K}$ observed in oxidation studies on polycrystalline SnSe [83].

With the aim of further tuning the carrier concentration, numerous dopants have been considered resulting in either $p$-type or $n$-type electrical conduction. For $p$-type doping, the main elements known to effectively dope $\mathrm{PbTe}$ and PbSe have been primarily investigated. This includes alkali metals (Na [83-89], K [85,87,90], Li [87]) and alkaline-earth elements (Ca, Sr and $\mathrm{Ba}$ [91]). In addition, various transition metals (Ag [82,92-95], Cu [91,96,97], Zn [91,98], Al [97], Tl [99], Ti [100] and In [97,101]), metalloids (Pb [89,97], Te [102104] and S [105,106]), Ge [91,107-109], as well as some lanthanides (Sm [110] and $\left.\mathrm{LaCl}_{3}[111]\right)$ have also been investigated. In contrast, the halogens $\mathrm{Cl}[112,113], \mathrm{Br}[114]$ and I [106] as well as $\mathrm{Bi}[113]$ were shown to induce $n$-type electrical conduction. Only few studies have used so far a multiple-doping approach on either a single site ( $n$-type $\mathrm{Sn}_{0.74} \mathrm{~Pb}_{0.20} \mathrm{Ti}_{0.06} \mathrm{Se}$ [100]) or on both sites (such as in $p$-type Sno.99Na0.01Se0.84Te0.16 [102]). In most cases, the introduction of the foreign element increases the carrier concentration which helps to optimize the power factor. Due to the low doping levels, most of these dopants have little influence on the lattice thermal conductivity, giving rise ultimately to enhanced $Z T$ values near $800 \mathrm{~K}$. So far, peak 
ZT values ranging between 0.8 and 1.3 have been achieved in either $p$-type or $n$-type samples.

The variations of the electronic properties with the carrier concentration, and more particularly, the evolution of the thermopower with the hole concentration has been successfully modelled by considering a simple parabolic band model assuming that either one or two valence bands govern the transport properties [87]. For this analysis, several sets of available data in the literature on both polycrystalline and single-crystalline samples have been considered (Figure 5).

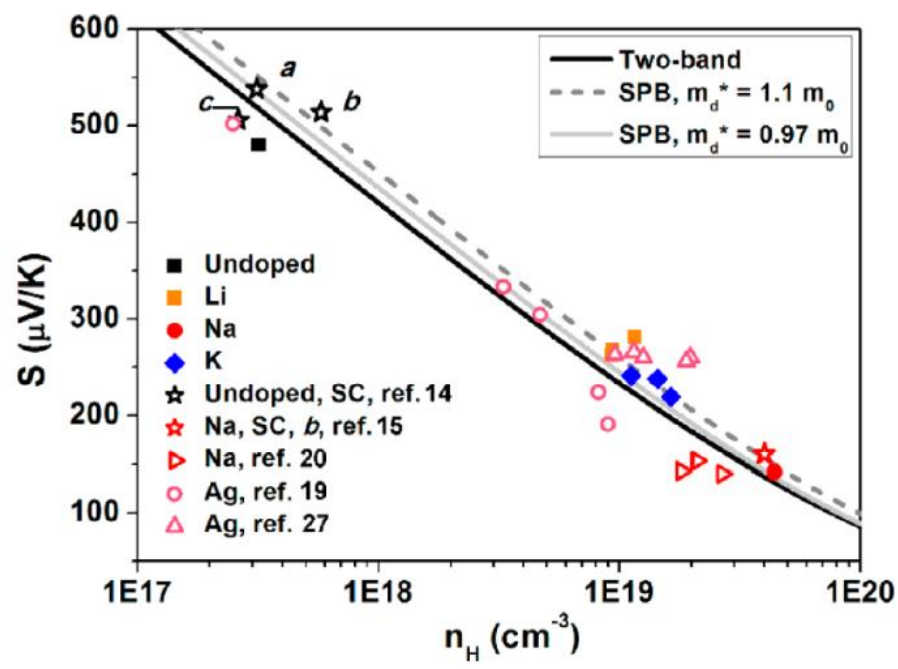

Fig. 5 Pisarenko-Ioffe plot (thermopower as a function of the hole concentration) at $300 \mathrm{~K}$.

Both data obtained on single-crystalline (SC) and polycrystalline doped SnSe have been added. A two-band model and two single-parabolic band (SPB) models with different density-of-states effective masses (denoted as $m_{d}^{*}$ ) have been considered to generate the black solid, grey dashed and grey solid curves, respectively. The $m_{d}^{*}$ value of 0.97 
corresponds to that determined theoretically and the value of 1.1 represents the best fit to the data. The references 14, 15, 19, 20 and 27 corresponds to the references [22], [38], [82], [84] and [94] in the present chapter, respectively. Reprinted (adapted) with permission from Wei et al. [87], J. Am. Chem. Soc. 138, 8875 (2016). Copyright 2016 by the American Chemical Society.

The average density-of-states effective mass $m_{D O S}^{*}$ used in transport equations can be calculated from the values along the three axes determined theoretically or experimentally by ARPES using the formula $m_{D O S}^{*}=\left(m_{a i}^{*} m_{b i}^{*} m_{c i}^{*}\right)^{1 / 3} \gamma^{2 / 3}$ (with the degeneracy factor $\gamma$ equal to 2 for SnSe). Each of these models (with one or two valence bands) describes equally well the variations in the thermopower with the hole concentration at $300 \mathrm{~K}$ for concentrations varying between $10^{17} \mathrm{~cm}^{-3}$ and $10^{20} \mathrm{~cm}^{-3}$ [87]. The best fit value of $m_{D O S}^{*}(1.1$ $m_{0}$ ) agrees very well with the value predicted by electronic band structure calculations or by ARPES experiments. These results consistently show that a single parabolic band model is sufficient to describe satisfactorily the evolution of the thermopower as a function of the doping level in $p$-type SnSe.

Further attempts at optimizing the thermoelectric properties of SnSe have been considered through the formation of solid solutions with SnS [105], SnTe [115] or PbSe $[116,117]$. Solid solutions are usually a convenient way to further control the carrier concentration and to decrease the lattice thermal conductivity via enhanced point defect scattering. Although this last mechanism is less pronounced in the present case due to the very low lattice thermal conductivity already exhibited by this compound, these solid 
solutions resulted in slightly enhanced ZT values with a peak value of 0.8 achieved at $823 \mathrm{~K}$

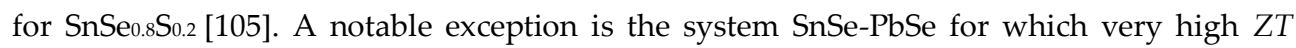
values of up to 1.7 at $873 \mathrm{~K}$ have been reported [116]. In this last study, the introduction of PbSe beyond the solubility limit led to the formation of nanoprecipitates, the influence of which on the transport properties has been proposed as the main key factor. However, the results reported in another study [117] on the same system show much lower values with a maximum twice as low as the above-mentioned value (around 0.85 at $800 \mathrm{~K}$ ). Further work seems therefore necessary to determine the reasons behind such significant discrepancy between the two studies.

Finally, some studies on composite samples have been reported recently where incorporation of $\mathrm{MoS}_{2}, \mathrm{MoSe} 2$ or graphene into SnSe has been studied [118,119]. However, none of them have led to a significantly higher ZT values compared to those achieved in pristine SnSe.

\section{Thermal properties}

\subsection{Specific heat of SnSe}

The specific heat $C_{p}$ provides valuable information on a given material below and above room temperature. At low temperatures, the specific heat represents an energyaveraged response that encodes the low-energy features of the phonon subsystem associated with optical phonons or zone-boundary excitations. At high temperatures, this 
thermodynamic property is an essential component to be measured accurately in order to correctly assess the thermal conductivity. The specific heat of SnSe has been studied on both polycrystalline and single-crystalline specimens (Figure 6).
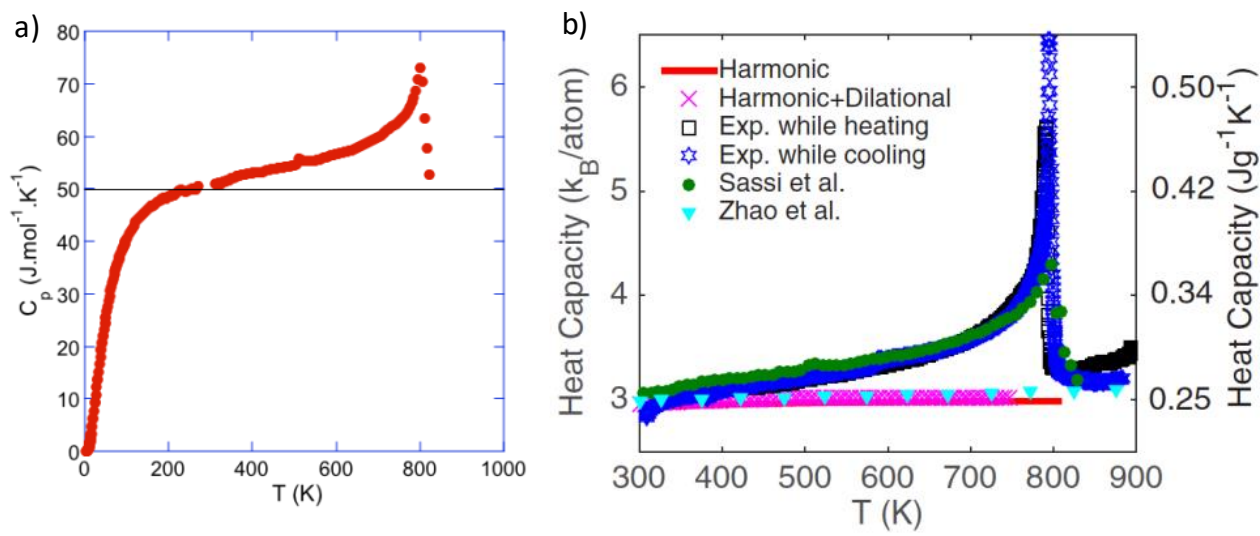

Fig. 6 (a) Temperature dependence of the specific heat of SnSe measured at low and high temperatures [120]. (b) High-temperature specific heat of SnSe determined experimentally by differential scanning calorimetry and calculated from the phonon density of states measured by inelastic neutron scattering. The data are compared to those shown in panel a) [120] and measured by Zhao et al. [22] on single crystals. (Panel a) Material reproduced from Sassi et al. [120], Materials Today: Proceedings 2, 690 (2015). (Panel b) Reproduced Figure 12 with permission from Bansal et al. [27]. Copyright 2016 by the American Physical Society.

DOI: 10.1103/PhysRevB.94.054307.

At low temperatures (Figure 6a), the results are consistent with the semiconducting ground state observed in pristine SnSe giving rise to a null electronic contribution to within 
experimental uncertainty $[120,121]$. Near $10 \mathrm{~K}$, the specific heat data, when plotted as $C_{p} / T^{3}$ versus $T$, deviate from a conventional Debye behaviour typified by a pronounced peak. This peak has been interpreted as either Einstein contributions or as a Schottky contribution $[120,121]$. In this last model [121], the origin of this peak has been discussed in terms of hard and soft substructures related to short and weaker bonds within the layers, respectively. These results thus show that the lattice dynamics of SnSe is dominated by two energy scales, in line with the two main regions observed in the phonon density of states $[44,66,122]$.

The temperature dependence of the specific heat at high temperatures steadily increases with increasing temperatures up to about $600 \mathrm{~K}$ (Figures $6 \mathrm{a}$ and $6 \mathrm{~b}$ ). Above this temperature, the nearly linear increase in $C_{p}$ turns into a non-linear variation. Near $800 \mathrm{~K}$, a clear peak is observed which corresponds to the orthorhombic-to-orthorhombic transition $[22,81,122]$. The transition appears as a broad peak between 650 up to $800 \mathrm{~K}$ in agreement with its continuous nature [22]. In order to determine the specific heat values over this temperature window for the calculation of the total thermal conductivity, it is therefore necessary to extrapolate linearly the values measured below $600 \mathrm{~K}$ up to $800 \mathrm{~K}$.

\subsection{Thermal conductivity of single-crystalline and polycrystalline SnSe}

One of the main ingredients that led to the very high $Z T$ values claimed in singlecrystalline SnSe is the very low lattice thermal conductivity values measured along the different axes of the crystal structure [22]. At $300 \mathrm{~K}$, the values were reported to be $0.46,0.70$ and $0.67 \mathrm{~W} \cdot \mathrm{m}^{-1} \cdot \mathrm{K}^{-1}$ which further decreases to reach $0.24,0.34$ and $0.31 \mathrm{~W} \cdot \mathrm{m}^{-1} \cdot \mathrm{K}^{-1}$ at $700 \mathrm{~K}$ 
along the $a, b$ and $c$ axes, respectively [22]. Of note, due to the high electrical resistivity values measured in pristine single crystals, the electronic contribution to the thermal conductivity $\kappa_{e}=L T / \rho$, where $L$ is the Lorenz number, is negligible so that $\kappa(T) \equiv \kappa_{L}(T)$.

Soon after this report, the question regarding the lattice thermal conductivity of single crystalline SnSe has rapidly become a central issue for several reasons. First, compared to the lattice thermal conductivity values measured in the 1960s [16-18], the values reported by Zhao et al. [22] are significantly lower. Second, the subsequent studies on the transport properties of polycrystalline pristine samples have reported higher lattice thermal conductivity values than in single crystals $[81,82]$. This result is counterintuitive since the presence of grain boundaries is expected to provide an additional phonon scattering channel which should lead to the opposite trend, that is, lower values should be observed in pristine polycrystalline samples. Third, the anisotropy observed between the three crystallographic axes was not following the sequence $\kappa_{L}^{a}<\kappa_{L}^{c}<\kappa_{L}^{b}$ predicted by $a b$ initio phonon calculations [123]. All these elements raised two fundamental questions: What could be the origin of such discrepancies between polycrystalline and single-crystalline samples? Were the values measured in single crystals by Zhao et al. [22] underestimated?

A first important point to answer these questions has been raised by Wei et al. [124] and is related to the density of the samples used in the study of Zhao et al. [22]. Using the thermal conductivity, the specific heat and the thermal diffusivity values reported, these authors pointed out that the density of the samples measured was corresponding to only $88 \%$ of the theoretical density of SnSe from crystallographic data [124]. This lower density could provide a natural explanation of the lower values measured in [22] and would thus 
point to a significant underestimation of the lattice thermal conductivity of single-crystalline SnSe. With higher $\kappa_{L}$ values, the contradiction between the values measured in polycrystalline and single-polycrystalline samples would then vanish, thereby recovering a more conventional trend in $\kappa_{L}$ from single to polycrystals.

Beyond this simplest explanation, several mechanisms have been advanced to try to explain the difference between single-crystalline and polycrystalline specimens as well as the lower values measured in the former [125]. Notably, air sensitivity under ambient conditions has been evoked as a possible problem. However, several studies have pointed out that SnSe is not air sensitive enough to consistently explain the observed differences in $\kappa_{L}$. Another possible reason discussed is the presence of "vast off-stoichiometry" in the single crystals studied by Zhao et al $[22,45]$. Since SnSe inherently possesses defects in the form of Sn vacancies, large amounts of such defects would tend to decrease the density in comparison to more stoichiometric crystals and would act as an efficient source of phonon scattering leading to lower $\kappa_{L}$ values. However, the estimated concentration of Sn vacancies should be as large as $16.5 \%$ to reconcile these different aspects $[45,124]$. This concentration is much higher than those obtained on single crystals grown under various synthetic conditions in the 1960s [16-18] and it remains unclear whether the structure of SnSe can accommodate such amounts, or equivalently, whether SnSe remains stable with such offstoichiometry.

Another point of concern related to this explanation is rooted in the band gap measured on these single crystals. Assuming that such large amounts of defects are indeed possible, we may expect these to have a significant influence on the electronic band 
structure. In particular, a significant variation in the band gap could be anticipated. However, direct measurements of the band gap width by absorption spectroscopy $[22,54]$ yielded results consistent with a band gap of around $0.9 \mathrm{eV}$ expected for stoichiometric SnSe. Both results could perhaps still be reconciled but this would require a rather surprising set of circumstances since the large amount of defects should indeed result in modifications of the electronic band structure but with a net result of a constant band gap. Of note, investigations on the influence of off-stoichiometry in polycrystalline Sn1-xSe (with $x$ $=0.01,0.02$ and 0.04$)$ are in agreement with the binary Sn-Se phase diagram indicating that the initial Sn-deficiency is accommodated by the formation of the secondary phase SnSez rather than by creating Sn vacancies [126]. Hence, whether significant Sn deficiency is possible in SnSe remains to be demonstrated and would require further theoretical and experimental investigations. In particular, calculations of the electronic band structure with large amounts of Sn vacancies should provide insights into the expected band structure. Moreover, systematic measurements of the band gap by absorption spectroscopy should be performed on single crystals with different carrier concentrations to determine whether varying the concentration of defects indeed can lead to modifications of the band gap.

Recent studies on pristine single-crystalline SnSe with densities close to the theoretical density for stoichiometric SnSe have been reported. These investigations have found lattice thermal conductivity higher than those reported by Zhao et al [22]. For instance, Ibrahim et al. [42] measured $\kappa_{L}$ values of $1.2,2.3$ and $1.7 \mathrm{~W} \cdot \mathrm{m}^{-1} \cdot \mathrm{K}^{-1}$ at $300 \mathrm{~K}$ along the $a, b$ and $c$ directions (Figure 7). 


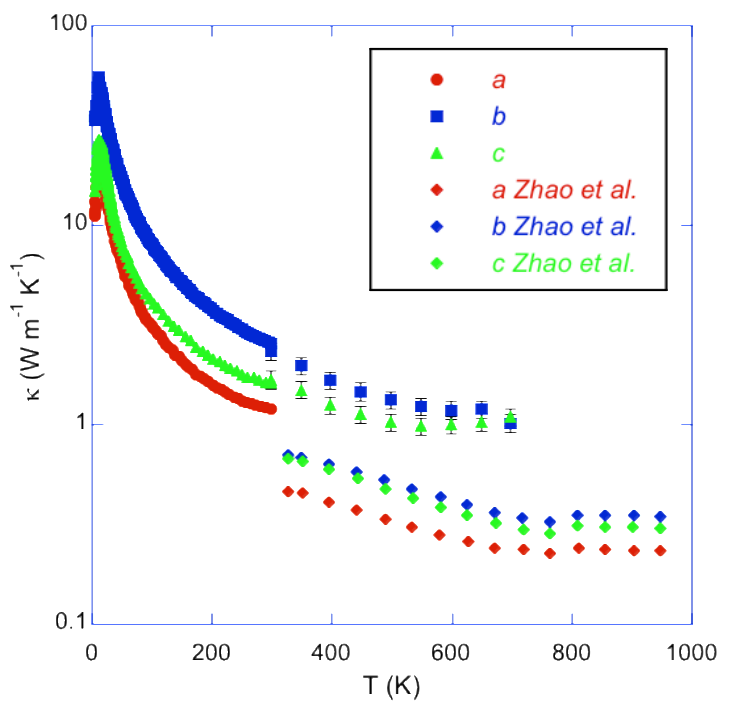

Fig. 7 Temperature dependence of the lattice thermal conductivity measured on singlecrystalline SnSe along the $a, b$ and $c$ directions [42]. The data are compared to those reported by Zhao et al. [22].

These values, between two and three times higher than those reported in prior studies, are consistent with early reports in the 1960s and with the sequence predicted by abinitio calculations $[16-18,123]$. Upon warming, these values decrease to reach a value of 1 W. $\mathrm{m}^{-1} \cdot \mathrm{K}^{-1}$ at $700 \mathrm{~K}$ in the $b$ and $c$ directions. As a consistency check, the values obtained by low-temperature measurements match very well with those obtained by a laser flash technique at high temperatures [42]. Since these measurements were carried out using a different measurement technology, it avoids any bias related to a particular technology.

Below $300 \mathrm{~K}, \kappa_{L}$ exhibits a crystalline-like behaviour with a pronounced Umklapp peak (also referred to as a dielectric maximum) that reaches 52,27 and $20 \mathrm{~W} \cdot \mathrm{m}^{-1} \cdot \mathrm{K}^{-1}$ at $15 \mathrm{~K}$ 
along the $b, c$ and $a$ directions, respectively (see Figure 7) [42,127]. Because the magnitude of this peak is sensitive to crystal imperfections, it usually can be considered as an effective metric of the quality of the grown crystal. The amplitude of this peak follows the sequence seen in the $\kappa_{L}$ values with the lowest values observed along the $a$ axis [42]. This result is not surprising since this direction corresponds to the interlayer direction along which the phonon mean free path is expected to be the shortest due to a stronger phonon scattering at the interface between the layers.

Another recent study on Na-doped and Ag-doped single crystals further corroborate all these results [127]. The measurements of the thermal conductivity at low temperatures is consistent with the above-mentioned main features. In particular, the Umklapp peak reported along the $b$ axis for pristine crystal is similar to that measured by Ibrahim et al. [42]. Upon doping, the amplitude of this peak is reduced due to additional point defect scattering induced by the doping atoms. What makes these results particularly interesting is that these measurements have been performed on crystals for which very high $Z T$ values had been reported at high temperatures (with a maximum ZT of 2 at $800 \mathrm{~K}$ [43]). This astonishing difference between both studies has been discussed in terms of inhomogeneous distribution of dopants in the crystals. Such discrepancy clearly warrants further experimental investigations to determine whether the $Z T$ values of up to 2 reported [43] are really achievable in SnSe or are rather due to an erroneous combination of transport properties measured on samples with different hole concentrations due to the inhomogeneous distribution of the doping species. 
In polycrystalline SnSe, measurements need to be performed both parallel and perpendicular to the pressing direction due to the anisotropic crystal structure $[81,82]$. Compared to single crystals, the literature data on polycrystals exhibit less dispersion and most of these studies agree on the values in pristine SnSe. At $300 \mathrm{~K}$, the measured $\kappa_{L}$ values are 0.9 and $1.4 \mathrm{~W} \cdot \mathrm{m}^{-1} \cdot \mathrm{K}^{-1}$ in the parallel and perpendicular direction, respectively $[81,82]$. The large Umklapp peak observed in single crystals survive in polycrystals with respective peak values of 12 and $6 \mathrm{~W} \cdot \mathrm{m}^{-1} \cdot \mathrm{K}^{-1}$ near $25 \mathrm{~K}$ [120]. Upon increasing temperature, the $\kappa_{L}$ values decrease to reach very low values that are close to $0.5 \mathrm{~W} \cdot \mathrm{m}^{-1} \cdot \mathrm{K}^{-1}$ in both directions (Figure 8).

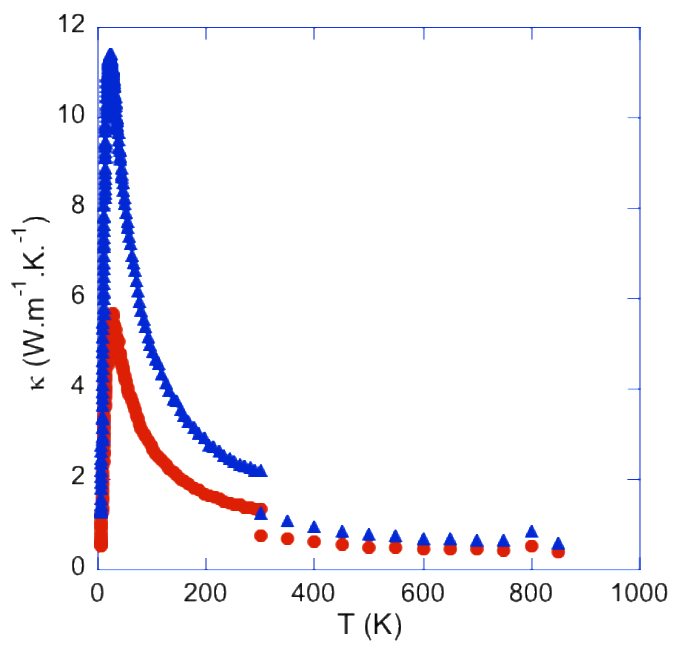

Fig. 8 Lattice thermal conductivity as a function of temperature measured on pristine polycrystalline SnSe parallel (circle symbol) and perpendicular (triangle symbol) to the pressing direction [120]. The difference observed near room temperature is due to thermal radiations present in low-temperature measurements. Material reproduced from Sassi et al.

[120], Materials Today: Proceedings 2, 690 (2015). 
One important aspect when estimating the lattice thermal conductivity of doped polycrystalline samples is related to the determination of the Lorenz number $L$ whose values sensitively depend on temperature and on the scattering mechanism. The $L$ values can thus significantly deviate at high temperatures from the conventional degenerate limit of the free electron or hole gas $\left(2.45 \times 10^{-8} \mathrm{~V}^{2} \cdot \mathrm{K}^{-2}\right)$. As mentioned earlier in this chapter, a single parabolic band model is sufficient to explain the trends in the electronic properties with the carrier concentration. This model can be also used to compute the temperature dependence of the Lorenz number in doped samples. This approach typically leads to Lorenz numbers that fall within the range $1.5 \times 10^{-8}-2 \times 10^{-8} \mathrm{~V}^{2} \cdot \mathrm{K}^{-2}$ at high temperatures $[87,127]$. When subtracting the electronic contribution with these $L$ values, similar $\kappa_{L}$ values are then observed in doped compounds regardless of the element used for doping. Some differences can nevertheless be observed since solid solutions or heavy doping can provide an additional phonon scattering source $[105,116,117]$.

\subsection{Microscopic origin of the low lattice thermal conductivity of SnSe}

Thanks to the possibility to grow large, high-quality single crystals of SnSe, advanced spectroscopic tools can be used to pinpoint the microscopic origin of the very low lattice thermal conductivity of this compound. To this end, inelastic neutron scattering is the experiment of choice since this allows to directly probe the acoustic and optical phonon dispersions. Such experiment carried out on SnSe has revealed several striking features that make it distinct from other rock-salt semiconductors $[44,122]$. These results have shown that 
the continuous structural phase transition near $800 \mathrm{~K}$ is associated with the condensation of a phonon mode that strongly softens upon warming (Figure 9).

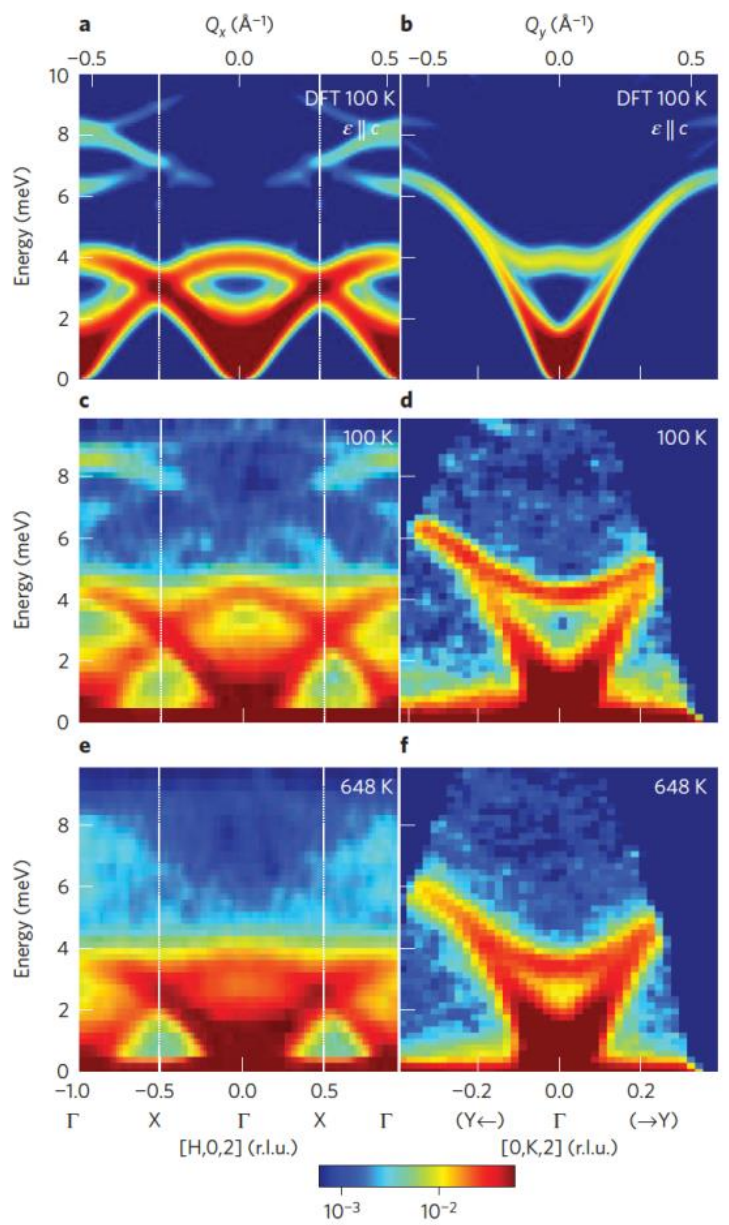

Fig. 9 Dynamical structure factors calculated by DFT at $100 \mathrm{~K}$ (panels a and b) and measured experimentally on single-crystalline SnSe at $100 \mathrm{~K}$ (panels c and d) and at $648 \mathrm{~K}$ (panels e and $\mathrm{f}$ ) along the $\Gamma-\mathrm{X}$ and $\Gamma-\mathrm{Y}$ directions. These measurements show the strong softening experienced by the low-energy optic phonons upon warming. Data reproduced from Li et al.

[44] Nat. Phys. 11, 1063 (2015). 
Intriguingly, this mode remains strongly anharmonic below the temperature of the phase transition over a large temperature window. This anharmonicity, beyond that associated with the conventional thermal expansion of the unit cell, gives rise to anharmonic phonon-phonon interactions which effectively scatter the heat-carrying acoustic phonons and naturally leads to the very low lattice thermal conductivity values measured. This type of lattice-instability-driven suppression of the heat transport is reminiscent to those seen in the related rock-salt semiconductors $\mathrm{PbTe}$, $\mathrm{SnTe}$ and GeTe [13-15]. However, some important differences exist between these three compounds and $\mathrm{SnSe}$. PbTe is close to a ferroelectric transition but its crystal structure remains stable due to the fact that the softening of the transverse optic mode is only partial [13-15]. As for both SnTe and GeTe, they exhibit a displacive distortion of their cubic crystal structure giving rise to a ferroelectric rhombohedral structure while SnSe is not ferroelectric in its low-temperature orthorhombic phase. SnSe thus represents a model system to study in more detail the role of this type of lattice instability and its related anharmonicity on the thermal transport.

\section{Conclusion}

The simple orthorhombic semiconductor SnSe has focused attention over the last years owing to the record-breaking $Z T$ values at high temperatures reported in Bridgmangrown single crystals. This announcement has revitalized the interest for this compound to gain an in-depth understanding of its structural, chemical and physical properties. Thanks to the presence of multivalleys in the valence bands revealed by various electronic band 
structure calculations and confirmed by ARPES experiments, SnSe exhibits good electronic transport properties near $800 \mathrm{~K}$. Combined with very low lattice thermal conductivity due to some peculiarities in its bonding scheme, this results in thermoelectric performances that rival those achieved in PbTe-based thermoelectric materials.

Several extrinsic dopants have been successfully used to tailor its thermoelectric properties revealing that $\mathrm{SnSe}$ is in fact as chemically flexible as other rock-salt semiconductors. These investigations have raised the $Z T$ values from 0.5 at $800 \mathrm{~K}$ in pristine samples up to around 1.0 at $800 \mathrm{~K}$ in properly-optimized polycrystalline samples. These lower values with respect to those announced in single crystals come from the significant difference in the lattice thermal conductivity measured. The fact that higher values have been measured in polycrystals challenges the usual trend that single crystals should possess higher lattice thermal conductivity due to the absence of grain boundary scattering. This counterintuitive finding has raised concern about the true lattice thermal conductivity values of single crystalline SnSe, an issue which is still debated at the time of writing this review.

As a concluding remark, does the (re)discovery of SnSe be considered as a breakthrough? On one hand, the announcement of record-breaking $Z T$ values has led to a plethora of theoretical and experimental investigations of its physical properties, should it be in bulk form or as thin film down to only one single layer. In this sense, our knowledge on this so far largely overlooked material has been significantly advanced over the last years thanks to the modern techniques employed to probe its basic properties. On the other hand, the lattice thermal conductivity values required to reach these high $Z T$ values seem to have 
been significantly underestimated, casting serious doubt on the possibility to experimentally achieve such high thermoelectric performances. Thus, the honorific title "best thermoelectric material ever discovered" that SnSe is sometimes dubbed is likely overemphasized. Although very intriguing on its own, this simple binary compound probably does not represent a real breakthrough in thermoelectricity. Yet, this compound as well as its Te- and S-based analogues remain a fascinating area of research to explore in years to come.

\section{References}

[1] H. J. Goldsmid Thermoelectric Refrigeration. Temple Press Books Ltd: London; 1964. DOI:10.1007/978-1-4899-5723-8.

[2] D. M. Rowe (ed.), Thermoelectrics and its Energy Harvesting (CRC Press, 2012).

[3] G. Tan, L.-D. Zhao, M. G. Kanatzidis, Chem. Rev. 116, 12123 (2016).

[4] T. Takabatake, K. Suekuni, T. Nakayama, E. Kaneshita, Re. Mod. Phys. 86, 669 (2014).

[5] M. M. Koza, H. Mutka, Y. Okamoto, J. Yamaura, Z. Hiroi, Phys. Chem. Chem. Phys. 17, 24837 (2015).

[6] D. J. Safarik, T. Klimczuk, A. Llobet, D. D. Byler, J. C. Lashley, J. R. O’Brien, N. R. Dilley, Phys. Rev. B 85, 014103 (2012).

[7] M. D. Nielsen, V. Ozolins, J. P. Heremans, Energy. Environ. Sci. 6, 570 (2013).

[8] Y. Bouyrie, C. Candolfi, S. Pailhès, M. M. Koza, B. Malaman, A. Dauscher, J. Tobola, O. Boisron, L. Saviot, B. Lenoir, Phys. Chem. Chem. Phys. 17, 19751 (2015). 
[9] J. Androulakis, I. Todorov, J. He, D. Y. Chung, V. Dravid, M. G. Kanatzidis, J. Am. Chem. Soc. 133,10920 (2011).

[10] L.-D. Zhao, V. P. Dravid, M. G. Kanatzidis Energy Environ. Sci. 7, 251 (2014).

[11] Z.-G. Chen, G. Han, L. Yang, L. Cheng, J. Zou, Prog. Nat. Sci.: Mater. Int. 22, 535 (2012).

[12] J.-F. Li, W.-S. Liu, L.-D. Zhao, M. Zhou, NPG Asia Mater. 2, 152 (2010).

[13] O. Delaire, J. Ma, K. Marty, A. F. May, M. A. McGuire, M.-H. Du, D. J. Singh, A. Podlesnyak, G. Ehlers, M. D. Lumsden, B. C. Sales, Nat. Mater. 10, 614 (2011).

[14] C. W. Li, J. Ma, H. B. Cao, A. F. May, D. L. Abernathy, G. Ehlers, C. Hoffmann, X. Wang, T. Hong, A. Huq, O. Gourdon, O. Delaire, Phys. Rev. B 90, 214303 (2014).

[15] C. W. Li, O. Hellman, J. Ma, A. F. May, H. B. Cao, X. Chen, A. D. Christianson, G. Ehlers, D. J. Singh, B. C. Sales, O. Delaire, Phys. Rev. Lett. 112, 175501 (2014).

[16] J. D. Wasscher, W. Albers, C. Haas, Solid State Electron. 6, 261 (1963).

[17] H. Maier, D. R. Daniel, J. Electron. Mater. 6, 693 (1977).

[18] W. Albers, C. Haas, H. Ober, G. R. Schodder, J. D. Wasscher, J. Phys. Chem. Solids 23, 215 (1962).

[19] M. J. Peters, L. E. McNeil, Phys. Rev. B 41, 5893 (1990).

[20] R. Sharma, Y. Chang, J. Phase Equilib. 7, 68 (1986).

[21] H. Okamoto, J. Phase Equilib. 19, 293 (1998).

[22] L.-D. Zhao, S.-H. Lo, Y. Zhang, H. Sun, G. Tan, C. Uher, C. Wolverton, V. P. Dravid, M.

G. Kanatzidis, Nature 508, 373 (2014).

[23] J. P. Heremans, Nature 508, 327 (2014).

[24] Z.-G. Chen, X. Shi, L.-D. Zhao, J. Zou, Prog. Mater. Sci. 97, 283 (2018). 
[25] R. Moshwan, L. Yang, J. Zou, Z.-G. Chen, Adv. Funct. Mater. 1703278 (2017).

[26] W. Li, Y. Wu, S. Lin, Z. Chen, J. Li, X. Zhang, L. Zheng, Y. Pei, ACS Energy Lett. 2, 2349 (2017).

[27] I. Pletikosić, F. von Rohr, P. Pervan, P. K. Das, I. Vobornik, R. J. Cava, T. Valla, Phys. Rev. Lett. 120, 156403 (2018).

[28] M. Sist, J. Zhang, B. Brummerstedt Iversen, Acta Crystallogr B 72, 310 (2016).

[29] J. J. Yan, F. Ke, C. L. Liu, L. Wang, Q. L. Wang, J. K. Zhang, G. H. Li, Y. H. Han, Y. Z. Ma, C. X. Gao, Phys. Chem. Chem. Phys. 18, 5012 (2016).

[30] T. Chattopadhyay, A. Werner, H. G. von Schnering, J. Pannetier, Rev. Phys. Appl. (Paris) 19, 807 (1984).

[31] I. Loa, R. J. Husband, R. A. Downie, S. R. Popuri, and J.-W. G. Bos, J. Phys.: Condens. Matter 27, 072202 (2015).

[32] J. Zhang, H. Y. Zhu, X. X. Wu, H. Cui, D. M. Li, J. R. Jiang, C. X. Gao, Q. S. Wang, Q. L. Cui, Nanoscale 7, 10807 (2015).

[33] S. M. de Souza, H. O. da Frota, D. M. Trichês, A. Ghosh, P. Chaudhuri, M. S. dos Santos Gusmao, A. F. F. de Figueiredo Pereira, M. C. Siqueira, K. D. Machado, J. C. de Lima, J. Appl. Cryst. 49, 213 (2016).

[34] S. Alptekin, J. Mol. Model 17, 2989 (2011).

[35] L. Makinistian and E. A. Albanesi, Comput.Mater. Sci. 50, 2872 (2011).

[36] Yu. A. Timofeev, B. V. Vinogradov, V. B. Begoulev, Phys. Solid State 39, 207 (1997).

[37] Xuliang Chen, Pengchao Lu, Xuefei Wang, Yonghui Zhou, Chao An, Ying Zhou, Cong Xian, Hao Gao, Zhaopeng Guo, Changyong Park, Binyang Hou, Kunling Peng, Xiaoyuan 
Zhou, Jian Sun, Yimin Xiong, Zhaorong Yang, Dingyu Xing, Yuheng Zhang, Phys. Rev. B 96, 165123 (2017).

[38] L.-D. Zhao, G. Tan, S. Hao, J. He, Y. Pei, H. Chi, H. Wang, S. Gong, H. Xu, V. P. Dravid, C. Uher, G. J. Snyder, C. Wolverton, M. G. Kanatzidis, Science 351, 141 (2016).

[39] C. Julien, M. Eddrief, I. Samaras, M. Balkanski, Mater. Sci. Eng. B-Adv. 15, 70 (1992).

[40] A. Elkorashy A, J. Phys. Chem. Solids 51, 289 (1990).

[41] A. Elkorashy, J. Phys. Chem. Solids 47, 497 (1986).

[42] D. Ibrahim, J.-B. Vaney, S. Sassi, C. Candolfi, V. Ohorodniichuk, P. Levinsky, C. Semprimoschnig, A. Dauscher, B. Lenoir, Appl. Phys. Lett. 110, 032103 (2017).

[43] K. Peng, X. Lu, H. Zhan, S. Hui, X. Tang, G. Wang, J. Dai, C. Uher, G. Wang, X. Zhou, Energy Environ. Sci. 9, 454 (2016).

[44] C. W.Li, J. Hong, A. F. May, D. Bansal, S. Chi, T. Hong, G. Ehlers, O. Delaire, Nat. Phys. 11,1063 (2015).

[45] D. Wu, L. Wu, D. He, L. D. Zhao, W. Li, M. Wu, M. Jin, J. Xu, J. Jiang, L. Huang, Y. Zhu, M. G. Kanatzidis, J. He, Nano Energy 35, 321 (2017).

[46] M. Jin, H. Shao, H. Hu, D. Li, J. Xu, G. Liu, J. Cryst. Growth 460, 112 (2017).

[47] M. Jin, H. Shao, H. Hu, D. Li, H. Shen, J. Xu, J. Alloy Compd. 712, 857 (2017).

[48] M. Nassary, Turk. J. Phys. 33, 201 (2009).

[49] V. Bhatt, K. Gireesan, G. Pandya, J. Cryst. Growth 96, 649 (1989).

[50] B. Nariya, A. Dasadia, M. Bhayani, A. Patel, A. Jani, Chalcogenide Lett. 6, 549 (2009).

[51] A. Agarwal, S. H. Chaki, D. Lakshminarayana, Mater. Lett. 61, 5188 (2007). 
[52] A. Agarwal, M. Vashi, D. Lakshminarayana, N. Batra, J. Mater. Sci-Mater. El 11, 67 (2000).

[53] A. Agarwal, P. Patel, D. Lakshminarayana, J. Cryst. Growth 142, 344 (1994).

[54] J. G. Yu, A. Yue, O. Stafsudd, J. Cryst. Growth 54, 248 (1981).

[55] M. Hong, Z.-G. Chen, L. Yang, T. C. Chasapis, S. D. Kang, Y. Zou, J. Mater. Chem. A 5, 10713 (2017).

[56] J. Guo, J. Jian, J. Liu, B. Cao, R. Lei, Z. Zhang, Nano Energy 38, 569 (2017).

[57] Y. Huang, C. Wang, X. Chen, D. Zhou, J. Du, S. Wang, L. Ning, RSC Adv. 7, 27612 (2017).

[58] Z. Wang, C. Fan, Z. Shen, C. Hua, Y. Hu, F. Sheng, Y. Lu, H. Fang, Z. Qiu, J. Lu, Z. Liu, W. Liu, Y. Huang, Z.-A. Xu, D. W. Shen, Y. Zheng, Nat. Commun. 9, 47 (2018).

[59] G. Shi, E. Kioupakis, J. Appl. Phys. 117, 065103 (2015).

[60] K. Kutorasinski, B. Wiendlocha, S. Kaprzyk, J. Tobola, Phys Rev B 91, 205201 (2015).

[61] Y. Suzuki, H. Nakamura, Phys. Chem. Chem. Phys. 17, 29647 (2015).

[62] S. Chen, K. Cai, W. Zhao, Phys. Rev. B, 407, 4154 (2012).

[63] R. L. González-Romero, A. Antonelli, J. J. Meléndez, Phys. Chem. Chem. Phys. 19, 12804 (2017).

[64] Y. Huang, L. Li, Y.-H. Lin, C.-W. Nan, J. Phys. Chem. C 121, 17530 (2017).

[65] A. Shafique, Y.-H. Shin, Sci. Rep. 7, 506 (2017).

[66] R. Guo, X. Wang, Y. Kuang, B. Huang, Phys. Rev. B 92, 115202 (2015).

[67] X. Guan, P. Lu, L. Wu, L. Han, G. Liu, Y. Song, S. Wang, J. Alloy Compd. 643, 116 (2015). 
[68] D. D. Cuong, S. H. Rhim, J.-H. Lee, S. C. Hong, AIP Advances 5, 117147 (2015).

[69] K. Tyagi, B. Gahtori, S. Bathula, N. K. Singh, S. Bishnoi, S. Auluck, A. K. Srivastava, A. Dhar, RSC Adv. 6, 11562 (2016).

[70] J. Yang, G. Zhang, G. Yang, C. Wang, Y. X. Wang, J. Alloy Compd. 644, 615 (2015).

[71] G. Ding, G. Gao, K. Yao, Sci. Rep. 5, 9567 (2015).

[72] A. J. Hong, L. Li, H. X. Zhu, Z. B. Yan, J. M. Liu, Z. F. Ren, J. Mater. Chem. A 3, 13365 (2015).

[73] K. Kuroki, R. Arita, J. Phys. Soc. Jpn. 76, 08370 (2007).

[74] K. Zhang, K. Deng, J. Li, H. Zhang, W. Yao, J. Denlinger, Y. Wu, W. Duan, S. Zhou, Phys. Rev Mater. 2, 054603 (2018).

[75] V. Tayari, B. V. Senkovskiy, D. Rybkovskiy, N. Ehlen, A. Fedorov, C.-Y. Chen, J. Avila, M. Asensio, A. Perucchi, P. di Pietro, L. Yashina, I. Fakih, N. Hemsworth, M. Petrescu, G. Gervais, A. Grüneis, T. Szkopek, Phys. Rev. B 97, 045424 (2018).

[76] Q. Lu, M. Wu, D. Wu, C. Chang, Y.-P. Guo, C.-S. Zhou, W. Li, X.-M. Ma, G. Wang, L.-D. Zhao, L. Huang, C. Liu, J. He, Phys. Rev. Lett. 119, 116401 (2017).

[77] D. M. Rowe (ed.), CRC Handbook of Thermoelectrics (CRC Press, London, 1995).

[78] A. T. Duong, G. D. Van Quang Nguyen, S. K. Van Thiet Duong, J. Y. Song, J. K. Lee, J. E. Lee, S. Park, T. Min, J. Lee, J. Kim, S. Cho, Nat. Commun. 7, 13713 (2016).

[79] T. M. H. Nguyen, A. T. Duong, G. Duvjir, T. L. Trinh, V. Q. Nguyen, J. Kim, S. Cho, Bull. Am. Phys. Soc. 62 (2017).

[80] T. T. Ly, G. Duvjir, T. Min, J. Buyn, T. Kim, M. M. Saad, N. T. M. Hai, S. Cho, J. Lee, J. Kim, Phys. Chem. Chem. Phys. 19, 21648 (2017). 
[81] S. Sassi, C. Candolfi, J.-B. Vaney, V. Ohorodniichuk, P. Masschelein, A. Dauscher, B. Lenoir, Appl. Phys. Lett. 104, 212105 (2014).

[82] C.-L. Chen, H. Wang, Y.-Y. Chen, T. Day, G. J. Snyder, J. Mater. Chem. A 2, 11171 (2014). [83] Y. Li, J. P. Heremans, J.-C. Zhao, J. Alloy Compnd. 669, 224 (2016).

[84] E. K. Chere, Q. Zhang, K. Dahal, F. Cao, J. Mao, and Z. Ren, J. Mater. Chem. A 4, 1848 (2016).

[85] Z.-H. Ge, D. Song, X. Chong, F. Zheng, L. Jin, X. Qian, L. Zheng, R. E. Dunin-Borkowski, P. Qin, J. Feng, L.-D. Zhao, J. Am. Chem. Soc. 139, 9714 (2017).

[86] H.-Q. Leng, M. Zhou, J. Zhao, Y.-M. Han, L.-F. Li, RSC Adv. 6, 9112 (2016).

[87] T. R. Wei, G. Tan, X. Zhang, C. F. Wu, J. F. Li, V. P. Dravid, G. J. Snyder, M. G. Kanatzidis, J. Am. Chem. Soc. 138, 8875 (2016).

[88] B. Cai, J. Li, H. Sun, P. Zhao, F. Yu, L. Zhang, D. Yu, Y. Tian, B. Xu, J Alloy Compd 727, 1014 (2017).

[89] Y. K. Lee, K. Ahn, J. Cha, C. Zhou, H. S. Kim, G. Choi, S. I. Chae, J.-H. Park, S.-P. Cho, S. H. Park, Y.-E. Sung, W. B. Lee, T. Hyeon, I. Chung, J. Am. Chem. Soc. 139, 10887 (2017). [90] Y.-X. Chen, Z.-H. Ge, M. Yin, D. Feng, X.-Q. Huang, W. Zhao, J. He, Adv. Funct. Mater. 26,6836 (2016).

[91] B. R. Ortiz, H. Peng, A. Lopez, P. A. Parilla, S. Lany, E. S. Toberer Phys. Chem. Chem. Phys. 17, 19410 (2015).

[92] L. Zhang, J. Wang, Q. Sun, P. Qin, Z. Cheng, Z. Ge, Z. Li, S. Dou, Adv. Energy Mater. 7, 1700573 (2017). 
[93] H. Guo, H. Xin, X. Qin, J. Zhang, D. Li, Y. Li, C. Song, C. Li, J Alloy Compd. 689, 87 (2016).

[94] H. Leng, M. Zhou, J. Zhao, Y. Han, L. Li, J. Electron. Mater. 45, 527 (2015).

[95] C.-H. Chien, C.-C. Chang, C.-L. Chen, C.-M. Tseng, Y.-R. Wu, M.-K. Wu, C.-H. Lee, Y.Y. Chen, RSC Adv. 7, 34300 (2017).

[96] N. K. Singh, S. Bathula, B. Gahtori, K. Tyagi, D. Haranath, A. Dhar, J. Alloy Compd. 668, 152 (2016).

[97] J. Gao, G. Xu, Intermetallics 89, 40 (2017).

[98] J. C. Li, D. Li, X. Y. Qin, J. Zhang, Scripta Mater. 126, 6 (2017).

[99] V. Kucek, T. Plechacek, P. Janicek, P. Ruleova, L. Benes, J. Navratil, C. Drasar, J. Electron. Mater. 45, 2943 (2016).

[100] F. Li, W. Wang, X. Qiu, Z.-H. Zheng, P. Fan, J. Luo, B. Li, Inorg. Chem. Front. 4, 1721 (2017).

[101] J. H. Kim, S. Oh, Y. M. Kim, H. S. So, H. Lee, J.-S. Rhyee, S.-D. Park, S.-J. Kim, J. Alloy Compd. 682, 785 (2016).

[102] T.-R. Wei, C.-F. Wu, X. Zhang, Q. Tan, L. Sun, Y. Pan, J.-F. Li, Phys. Chem. Chem. Phys. 17, 30102 (2015).

[103] M. Hong, Z.-G. Chen, L. Yang, T. C. Chasapis, S. D. Kang, Y. Zou, G. J. Auchterlonie, M. G. Kanatzidis, G. J. Snyder, J. Zou, J. Mater. Chem. A 5, 10713 (2017).

[104] S. Chen, K. Cai, W. Zhao Phys. B: Condens. Matter 407, 4154 (2012).

[105] Y.-M. Han, J. Zhao, M. Zhou, X.-X. Jiang, H.-Q. Leng, L.-F. Li, J. Mater. Chem. A 3, 4555 (2015). 
[106] Q. Zhang, E. K. Chere, J. Sun, F. Cao, K. Dahal, S. Chen, G. Chen, Z. Ren, Adv. Energy Mater. 5, 1500360 (2015).

[107] M. Gharsalla, F. Serrano-Sanchez, N. M. Nemes, F. J. Mompean, J. L. Martinez, M. T. Fernandez-Diaz, F. Elhalouani, J. A. Alonso, Sci. Rep. 6, 26774 (2016).

[108] Y. Fu, J. Xu, G.-Q. Liu, X. Tan, Z. Liu, X. Wang, H. Shao, H. Jiang, B. Liang, J. Electron. Mater. 46, 3182 (2017).

[109] J. Gao, Z. Shao, G. Xu, Int. J. Appl. Ceram. Tech. 14, 963 (2017).

[110] J. Gao, H. Zhu, T. Mao, L. Zhang, J. Di, G. Xu, Mater. Res. Bull. 93, 366 (2017).

[111] F. Li, W. Wang, Z.-H. Ge, Z. Zheng, J. Luo, P. Fan, B. Li, Materials 11, 203 (2018).

[112] G. Han, S. R. Popuri, H. F. Greer, L. F. Llin, J. W. G. Bos, W. Zhou, D. J. Paul, H. Ménard, A. R. Knox, A. Montecucco, J. Siviter, E. A. Man, W. Li, M. C. Paul, M. Gao, T. Sweet, R. freer, F. Azough, H. Baig, T. K. Mallick, D. H. Gregory, Adv. Energy Mater. 7, 1602328 (2017).

[113] X. Wang, J. Xu, G. Liu, Y. Fu, Z. Liu, X. Tan, H. Shao, H. Jiang, T. Tan, J. Jiang, Appl. Phys. Lett. 108, 083902 (2016).

[114] D. Li, X. Tan, J. Xu, G. Liu, M. Jin, H. Shao, H. Huang, J. Zhang, J. Jiang, RSC Adv. 7, 17906 (2017).

[115] S. Siol, A. Holder, B. R. Ortiz, P. A. Parilla, E. S. Toberer, S. Lany, A. Zakutayev, RSC Adv. 7, 24747 (2017).

[116] G. Tang, W. Wei, J. Zhang, Y. Li, X. Wang, G. Xu, C. Chang, Z. Wang, Y. Du, L.-D. Zhao, J. Am. Chem. Soc. 138, 13647 (2016). 
[117] T.-R. Wei, G. Tan, C.-F. Wu, C. Chang, L.-D. Zhao, J.-F. Li, Appl. Phys. Lett. 110, 053901 (2017).

[118] S. Yang, J. Si, Q. Su, H. Wu, Mater. Lett. 193, 146 (2017).

[119] X.-Q. Huang, Y.-X. Chen, M. Yin, D. Feng, J. He, Nanotechnology 28, 105708 (2017).

[120] S. Sassi, C. Candolfi, J.-B. Vaney, V. Ohorodniichuk, P. Masschelein, A. Dauscher, B. Lenoir, Materials Today: Proceedings 2, 690 (2015).

[121] S. R. Popuri, M. Pollet, R. Decourt, M. Viciu, J.-W. G. Bos, Appl. Phys. Lett. 110, 253903 (2017).

[122] D. Bansal, J. W. Hong, C. W. Li, A. F. May, W. Porter, M. Y. Hu, D. L. Abernathy, O. Delaire, Phys. Rev. B 94, 054307 (2016).

[123] J. Carrete, N. Mingo, S. Curtarolo, Appl. Phys. Lett. 105, 101907 (2014).

[124] P.-C. Wei, S. Bhattacharya, J. He, S. Neeleshwar, R. Podila, Y. Y. Chen, A. M. Rao, Nature 539, E1-2 (2016).

[125] L.-D. Zhao, C. Chang, G. Tan, M. G. Kanatzidis, Energy Environ. Sci. 9, 3044 (2016).

[126] S. T. Lee, M. J. Kim, G.-G. Lee, S. G. Kim, S. Lee, W.-S. Seo, Y. S. Lim, Current Appl. Phys. 17, 732 (2017).

[127] S. Wang, S. Hui, K. Peng, T. P. Bailey, W. Liu, Y. Yan, X. Zhou, X. Tang, C. Uher, Appl. Phys. Lett. 112, 142102 (2018). 